\title{
Circumferential wall enhancement in evolving intracranial aneurysms on magnetic resonance vessel wall imaging
}

\author{
Shunsuke Omodaka, MD, PhD, ${ }^{1}$ Hidenori Endo, MD, PhD, ${ }^{2}$ Kuniyasu Niizuma, MD, PhD, ${ }^{2}$ \\ Miki Fujimura, MD, PhD, ${ }^{1}$ Takashi Inoue, MD, PhD, ${ }^{3}$ Toshiki Endo, MD, PhD, ${ }^{1}$ \\ Kenichi Sato, MD, PhD, ${ }^{4}$ Shin-ichiro Sugiyama, MD, PhD, ${ }^{5}$ and Teiji Tominaga, MD, $\mathrm{PhD}{ }^{2}$
}

Departments of ${ }^{1}$ Neurosurgery, ${ }^{4}$ Neuroendovascular Therapy, and ${ }^{5}$ Neuroanesthesia, Kohnan Hospital; ${ }^{2}$ Department of Neurosurgery, Tohoku University Graduate School of Medicine; and ${ }^{3}$ Department of Neurosurgery, Sendai Medical Center, Sendai, Japan

\begin{abstract}
OBJECTIVE Recent MR vessel wall imaging studies have indicated intracranial aneurysms in the active state could show circumferential enhancement along the aneurysm wall (CEAW). While ruptured aneurysms frequently show CEAW, CEAW in unruptured aneurysms at the evolving state (i.e., growing or symptomatic) has not been studied in detail. The authors quantitatively assessed the degree of CEAW in evolving unruptured aneurysms by comparing it separately to that in stable unruptured and ruptured aneurysms.
\end{abstract}

METHODS A quantitative analysis of CEAW was performed in 26 consecutive evolving aneurysms using MR vessel wall imaging. Three-dimensional T1-weighted fast spin echo sequences were obtained before and after contrast media injection, and the contrast ratio of the aneurysm wall against the pituitary stalk $\left(\mathrm{CR}_{\text {stalk }}\right)$ was calculated as the indicator of CEAW. Aneurysm characteristics of evolving aneurysms were compared with those of 69 stable unruptured and 67 ruptured aneurysms.

RESULTS The $\mathrm{CR}_{\text {stalk }}$ values in evolving aneurysms were significantly higher than those in stable aneurysms $(0.54$ vs $0.34, p<0.0001)$, and lower than those in ruptured aneurysms $(0.54$ vs $0.83, p<0.0002)$. In multivariable analysis, $\mathrm{CR}_{\text {stalk }}$ remained significant when comparing evolving with stable aneurysms (odds ratio [OR] 12.23, 95\% confidence interval [Cl] 3.53-42.41), and with ruptured aneurysms (OR 0.083, 95\% Cl 0.022-0.310).

CONCLUSIONS The CEAW in evolving aneurysms was higher than those in stable aneurysms, and lower than those in ruptured aneurysms. The degree of CEAW may indicate the process leading to rupture of intracranial aneurysms, which can be useful additional information to determine an indication for surgical treatment of unruptured aneurysms.

https://thejns.org/doi/abs/10.3171/2018.5.JNS18322

KEYWORDS circumferential aneurysm wall enhancement; evolving intracranial aneurysm; growing; symptomatic; magnetic resonance vessel wall imaging; 3D T1-weighted fast spin echo; vascular disorders

$\mathrm{U}$ NRUPTURED intracranial aneurysms have a fairly high prevalence of approximately $3 \%$ in the general population, ${ }^{28,29}$ whereas the incidence of subarachnoid hemorrhage is substantially lower, estimated to be $10-30$ per 100,000 people per year. ${ }^{5,18,31}$ Identification of an unruptured aneurysm offers an opportunity for preventive microsurgical or endovascular occlusion, but both treatment methods carry risks of complications. ${ }^{28}$
Therefore, identification of patients at high risk for rupture is mandatory for the management of aneurysms, and improved diagnostic technology offers the hope of more accurate prediction of aneurysm rupture.

Evolving unruptured aneurysms, including aneurysms with growth and/or symptoms, are considered to have a large risk of rupture. ${ }^{2,3,8,12}$ Recently, the incidence and degree of circumferential enhancement along the aneurysm

ABBREVIATIONS $\mathrm{ACA}=$ anterior cerebral artery; $\mathrm{CEAW}=$ circumferential enhancement along the aneurysm wall; $\mathrm{Cl}=$ confidence interval; $\mathrm{CR}_{\text {stalk }}=$ contrast ratio of the aneurysm wall against the pituitary stalk; ICA = internal carotid artery; $M C A=$ middle cerebral artery; $\mathrm{OR}=$ odds ratio; $\mathrm{PCOA}=$ posterior communicating artery; $\mathrm{ROC}=$ receiver operating characteristic; $\mathrm{SI}$ = signal intensity; T1-FSE = T1-weighted fast spin echo; TOF = time of flight.

SUBMITTED February 3, 2018. ACCEPTED May 2, 2018.

INCLUDE WHEN CITING Published online October 19, 2018; DOI: 10.3171/2018.5.JNS18322. 
wall (CEAW) on MR vessel wall imaging has been described to be higher in ruptured intracranial aneurysms than in unruptured aneurysms. ${ }^{6,19,23}$ However, reports on CEAW in evolving unruptured aneurysms have been limited and only investigated as unstable aneurysms, including ruptured aneurysms. ${ }^{6}$ Evolving aneurysms can exhibit wall enhancement because wall enhancement reflects inflammation activity that is considered to play an important role in aneurysm evolution. The purpose of this study is to quantitatively investigate CEAW in evolving aneurysms by separately comparing it to those in stable unruptured and ruptured aneurysms.

\section{Methods}

\section{Study Population and Data Extraction}

This study was approved by the IRB of Kohnan Hospital. Aneurysm data were extracted from an institutional database, which includes consecutive patients with surgically and/or endovascularly treated intracranial aneurysms. We divided aneurysms into three groups: evolving, stable, and ruptured aneurysms. An unruptured aneurysm was considered to be evolving in cases in which it was growing or the patient was symptomatic (neurological deficit) at the time MR vessel wall imaging was acquired. General headache was not considered as a symptom. We defined aneurysm growth as 3 patterns on the basis of the MR angiography findings: 1) maximum aneurysm diameter increase by $2 \mathrm{~mm}$ or more, 2) appearance of bleb, or 3 ) de novo aneurysm formation. Unruptured aneurysms without evidence of evolving, with more than $1 \mathrm{MR}$ angiography assessment at intervals of at least 6 months, were defined as stable aneurysms. We included consecutive cases with evolving intracranial saccular aneurysms presenting between March 2014 and March 2017. Study exclusion criteria were: 1) a paraclinoid aneurysm, 2) an aneurysm smaller than $3 \mathrm{~mm}$ or larger than $12 \mathrm{~mm}$ in size, and 3) a previously treated aneurysm. For comparison, stable aneurysms and ruptured aneurysms treated between March 2014 and March 2017 were also included. For ruptured an- eurysms, only surgically treated aneurysms were included because the confirmation of rupture can be uncertain in endovascularly treated aneurysms. The same exclusion criteria mentioned above were applied to these stable unruptured and ruptured aneurysms.

\section{Imaging}

A 3-T or 1.5-T MRI machine (Signa HDxt, GE Healthcare) was used in this study. In addition to time-of-flight (TOF) MR angiography, MR vessel wall imaging, which consisted of the single-slab 3D T1-weighted fast spin echo (T1-FSE) sequence, ${ }^{20}$ was performed. The acquisition parameters are summarized in Table 1. Gadopentetate dimeglumine (Magnescope, Guerbet) was administered intravenously $(0.1 \mathrm{mmol} / \mathrm{kg})$, and the 3D T1-FSE sequence was repeated 5 minutes after the contrast material was administered. The voxel data were exported into a personal computer for intensity analysis. The aneurysm location was classified as the anterior cerebral artery (ACA), the posterior communicating artery $(\mathrm{PCoA})$, the internal carotid artery (ICA) other than the PCoA, the middle cerebral artery (MCA), and the posterior circulation. ${ }^{10}$ The maximum measurement of aneurysm diameter on rotational angiography or MR angiography was defined as the aneurysm size.

\section{Measurement of Aneurysm Wall Enhancement}

Multiplanar oblique reconstructions obtained from preand postcontrast 3D T1-FSE and 3D TOF imaging were analyzed after coregistration using Amira 6.4 (Thermo Fisher Scientific). The reviewer was blinded to the clinical data but aware of aneurysm location. The contrast ratio of the aneurysm wall against the pituitary stalk $\left(\mathrm{CR}_{\text {stalk }}\right)$ was used to characterize the extent of CEAW as described elsewhere. ${ }^{22,23}$ We defined the volume of interest with the highest signal intensity (SI) in the circumference of the aneurysm and those in the whole pituitary stalk on postcontrast imaging, and the averaged SI of the aneurysm circumferential wall $\left(\mathrm{SI}_{\text {wall }}\right)$ and pituitary stalk $\left(\mathrm{SI}_{\text {stalk }}\right)$ in each

TABLE 1. Magnetic resonance acquisition parameters of 3D TOF and T1-FSE sequences

\begin{tabular}{|c|c|c|c|c|}
\hline \multirow[b]{2}{*}{ Parameter } & \multicolumn{2}{|c|}{ TOF } & \multicolumn{2}{|c|}{ T1-FSE } \\
\hline & 1.5 Tesla & 3 Tesla & 1.5 Tesla & 3 Tesla \\
\hline No. of channels & 8 & 12 & 8 & 12 \\
\hline No. of slabs & 3 & 1 & 1 & 1 \\
\hline TR, msec & 29 & 25 & 500 & 600 \\
\hline TE, msec & 6.8 & 3.3 & Minimum & Minimum \\
\hline FOV, $\mathrm{cm}$ & 20 & 20 & 25.6 & 25.6 \\
\hline Matrix & $352 \times 160$ & $512 \times 224$ & $256 \times 256$ & $256 \times 256$ \\
\hline Voxel size, $\mathrm{mm}^{3}$ & $0.6 \times 1.3 \times 0.6$ & $0.4 \times 0.9 \times 0.5$ & $0.5 \times 0.5 \times 0.5^{*}$ & $0.5 \times 0.5 \times 0.5^{*}$ \\
\hline Slice thickness, mm & 1.2 & 1.0 & 1.0 & 1.0 \\
\hline Bandwidth, $\mathrm{kHz}$ & 19.2 & 41.7 & 83.3 & 62.5 \\
\hline Echo train length & - & - & 28 & 22 \\
\hline Scan time, min:sec & $4: 55$ & $4: 27$ & $3: 36$ & $3: 41$ \\
\hline
\end{tabular}


TABLE 2. Characteristics of evolving, ruptured, and stable intracranial aneurysms

\begin{tabular}{|c|c|c|c|c|c|}
\hline \multirow[b]{2}{*}{ Characteristic } & \multirow[b]{2}{*}{ Evolving $(n=26)$} & \multirow[b]{2}{*}{ Stable $(n=69)$} & \multirow[b]{2}{*}{ Ruptured ( $n=67$ ) } & \multicolumn{2}{|c|}{$p$ Value } \\
\hline & & & & Evolving vs Stable & Evolving vs Ruptured \\
\hline Age, yrs* & $70(34-81)$ & $65(41-80)$ & $62(33-87)$ & 0.118 & 0.017 \\
\hline Female, n (\%) & $25(96)$ & $49(71)$ & $47(70)$ & 0.011 & 0.006 \\
\hline Aneurysm location, n (\%) & & & & 0.676 & 0.800 \\
\hline ACAs & $6(23)$ & $9(13)$ & $20(30)$ & & \\
\hline PCoA & $8(31)$ & $17(25)$ & $19(28)$ & & \\
\hline ICA other than PCoA & $2(8)$ & $6(9)$ & $5(7)$ & & \\
\hline MCA & $9(35)$ & $33(48)$ & $17(25)$ & & \\
\hline Posterior circulation & $1(4)$ & $4(6)$ & $6(9)$ & & \\
\hline Aneurysm size, mm* & $5.5(3.2-11.4)$ & $4.9(3.0-7.5)$ & $5.8(3.0-10.2)$ & 0.051 & 0.687 \\
\hline $\mathrm{CR}_{\text {stalk }}{ }^{*}$ & $0.54(0.18-1.39)$ & $0.34(0.12-0.86)$ & $0.83(0.25-1.83)$ & $<0.0001$ & $<0.0002$ \\
\hline
\end{tabular}

* The data of continuous variables are given as median (range).

of the volumes of interest was measured. Then, the $\mathrm{CR}_{\text {stalk }}$ was calculated as $\mathrm{SI}_{\text {wall }} / \mathrm{SI}_{\text {stalk }}$. The detailed calculation procedure and excellent reproducibility of this parameter have been previously described..$^{22,23}$

\section{Statistical Analysis}

Aneurysm status was categorized as evolving, stable, or ruptured. Continuous variables are presented as median (range), and categorical variables are presented as a number and corresponding percentage. The characteristics of evolving aneurysms were compared separately with each of the two control groups. We assessed the difference in variables between groups using the Wilcoxon rank-sum test for continuous variables and the chi-square or Fisher exact test for categorical variables. The median value of each continuous variable was used as the cutoff value for multivariate analysis. Multivariate logistic regression analysis was performed to determine factors independently associated with evolving aneurysms, including variables that reached $p$ values $<0.2$ in the univariate analysis. A $p$ value $<0.05$ was considered to indicate a statistically significant difference. The cutoff value of $\mathrm{CR}_{\text {stalk }}$ with the best sensitivity and specificity for differentiating evolving from stable aneurysms was identified on receiver operating characteristic (ROC) curve analysis. All of the statistical analyses were performed with JMP Pro (version 13.1, SAS Institute Inc.).

\section{Results}

During the study period, a total of 51 evolving aneurysms were treated. Twenty-five aneurysms were excluded due to aneurysm diameters larger than $12 \mathrm{~mm}(\mathrm{n}=17)$, paraclinoid aneurysm $(n=4)$, or previously treated aneurysm $(n=4)$. We included 26 evolving aneurysms: 24 growing (increase in diameter more than $2 \mathrm{~mm}$ in 16, appearance of bleb in 6 , and de novo formation in 2 aneurysms) and 3 symptomatic aneurysms (1 overlapping). All 3 symptomatic aneurysms presented with acutely developing oculomotor nerve palsy caused by a PCoA aneurysm. In these symptomatic cases, there was no episode of sudden headache and no evidence of hemorrhage on preop- erative CT and MRI study, and 1 showed growth on MR angiography. We also included 69 stable unruptured and 67 ruptured aneurysms for comparison.

The results of the univariate analysis are summarized in Table 2. The $\mathrm{CR}_{\text {stalk }}$ in evolving aneurysms was significantly higher than stable $(0.54$ vs $0.34, p<0.0001)$ and lower than ruptured aneurysms $(0.54$ vs 0.83 , p $<0.0002$; Fig. 1). Among the other clinical variables, age was significantly higher in evolving than ruptured aneurysms, and female predominance was significantly higher in evolving aneurysms than in both stable and ruptured aneurysms.

The results of the multivariate analysis are summarized in Table $3 . \mathrm{CR}_{\text {stalk }}$ remained significant when comparing evolving with stable aneurysms (odds ratio [OR] 12.23, 95\% confidence interval [CI] 3.53-42.41), and with ruptured aneurysms (OR 0.083 , 95\% CI $0.022-0.310)$. On ROC curve analysis (Fig. 2), the optimal cutoff value of $\mathrm{CR}_{\text {stalk }}$ to distinguish evolving from stable aneurysms was 0.39 (areas under the curve $=0.80$ ). Using a cutoff value of 0.39 for $\mathrm{CR}_{\text {stalk }}$, the sensitivity and specificity were 0.88 and 0.63 , respectively. Representative cases are illustrated in Fig. 3.

\section{Discussion}

Our study showed that the degree of CEAW in evolving aneurysms is significantly higher than that in stable aneurysms and lower than that in ruptured aneurysms. Among other variables, older age and female sex were associated with evolving aneurysms, which is consistent with previous reports. ${ }^{1-3,15}$ To our knowledge, this is the first report that quantitatively analyzes CEAW in evolving unruptured aneurysms by using MR vessel wall imaging.

CEAW has been shown to be a characteristic of ruptured aneurysms using MR vessel wall imaging. ${ }^{6,19,21,23}$ The strong CEAW in ruptured aneurysms can be explained by the physical disruption of the aneurysm wall and/or the inflammatory healing process following the rupture event, in addition to the preexisting inflammatory activity prior to rupture. ${ }^{9}, 13,14,19$ Evolving unruptured aneurysms can also show CEAW because of their inflammatory activity, which is hypothesized to contribute to aneurysm progression to- 


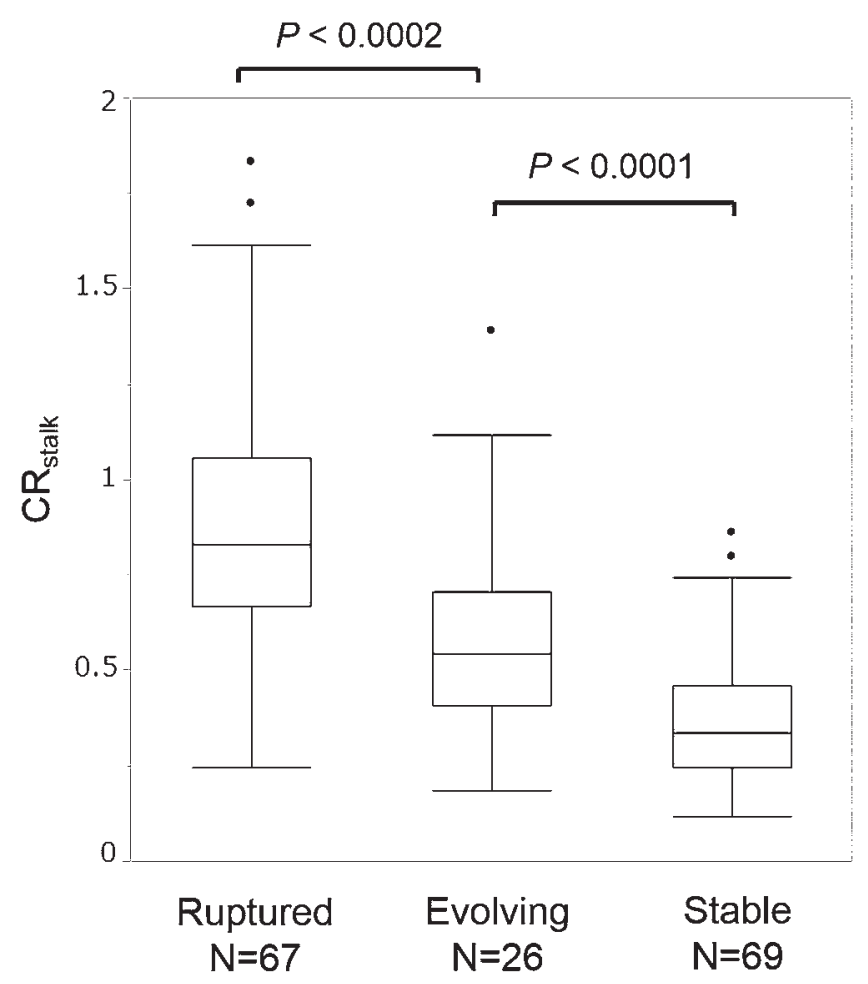

FIG. 1. Boxplots for the degree of aneurysm wall enhancement according to aneurysm status. The box signifies the upper and lower quartiles, and the median is represented by a line within the box. The whiskers are the largest and smallest values; values that differ by more than 1.5 -times the interquartile range from the median value are identified as outliers (black dots). The $\mathrm{CR}_{\text {stalk }}$ values in evolving aneurysms are significantly higher than those in stable aneurysms, and significantly lower than those in ruptured aneurysms.

ward rupture. ${ }^{6,11}$ In a study by Edjlali et al., the authors grouped ruptured and evolving unruptured aneurysms together as unstable aneurysms. ${ }^{6}$ They then compared the CEAW, which was assessed qualitatively, in the unstable aneurysms with those in stable (incidental and nonevolving) aneurysms, and the CEAW was more frequently observed in unstable than stable aneurysms (87\% vs $28.5 \%$, respectively). In the present study, we distinguished evolving aneurysms from ruptured aneurysms because ruptured and unruptured aneurysms differ in their essential structural pathology. ${ }^{13}$ In fact, ruptured aneurysms showed greater CEAW than evolving aneurysms, which indicated the effect of structural fragility in the ruptured aneurysms on CEAW.

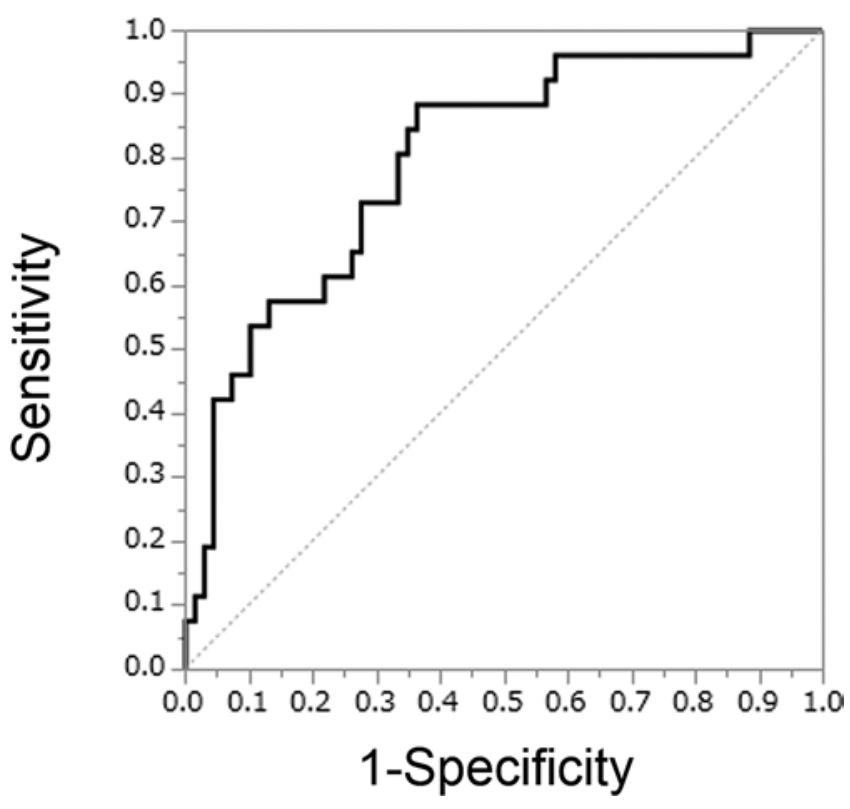

FIG. 2. ROC curve of the $\mathrm{CR}_{\text {stalk }}$ in differentiating evolving from stable aneurysms. The area under the curve is 0.80 .

Our study also showed that evolving aneurysms have greater CEAW than stable aneurysms. This result indicates the potential contribution of CEAW for the prediction of a patient's risk for a future rupture. According to the proposed model of the natural course of intracranial aneurysms, ${ }^{26,30}$ aneurysms may: 1) rupture immediately after aneurysm formation, 2) form and grow before rupture, and 3) remain stable for a long time after formation. These scenarios highlight the difficulty of accurately predicting the outcome after aneurysm formation, and the need for better surrogates for the outcome. Recent studies showed that the vulnerable plaques in the major intracranial arteries have a higher degree of contrast enhancement on MRI compared with stable plaques due to active inflammation in the vessel wall. ${ }^{17,25}$ Similarly, the evolving unruptured aneurysms with CEAW in this study might reflect the instability or vulnerability of the aneurysmal wall.

Inflammation of the aneurysm wall is increasingly considered to play a pivotal role in aneurysm progression., ${ }^{4,7}$ The inflammatory process, which is initiated by a hemodynamic insult, can result in destructive events in the aneurysm wall, including dysfunction of the endothelial cells and vascular smooth muscle cells and degradation of the extracellular matrix. These destructive events can trigger

TABLE 3. Multivariate logistic regression analysis for factors associated with evolving aneurysms

\begin{tabular}{lccccc}
\hline \multirow{2}{*}{ Factor } & \multicolumn{2}{c}{ Comparison w/ Stable } & & \multicolumn{2}{c}{ Comparison w/ Ruptured } \\
\cline { 2 - 3 } \cline { 5 - 6 } & p Value & OR $(95 \% \mathrm{Cl})$ & & $\mathrm{p} \mathrm{Value}$ & OR $(95 \% \mathrm{Cl})$ \\
\hline Age $>$ median & 0.6295 & $1.32(0.43-4.08)$ & & 0.0189 & $4.06(1.26-13.11)$ \\
\hline Female sex & 0.0171 & $14.18(1.60-125.43)$ & & 0.1084 & $5.96(0.67-52.79)$ \\
\hline Aneurysm size $>$ median & 0.9089 & $1.07(0.34-3.32)$ & & - & - \\
\hline $\mathrm{CR}_{\text {stalk }}>$ median & $<0.0001$ & $12.23(3.53-42.41)$ & & 0.0002 & $0.083(0.022-0.310)$ \\
\hline
\end{tabular}




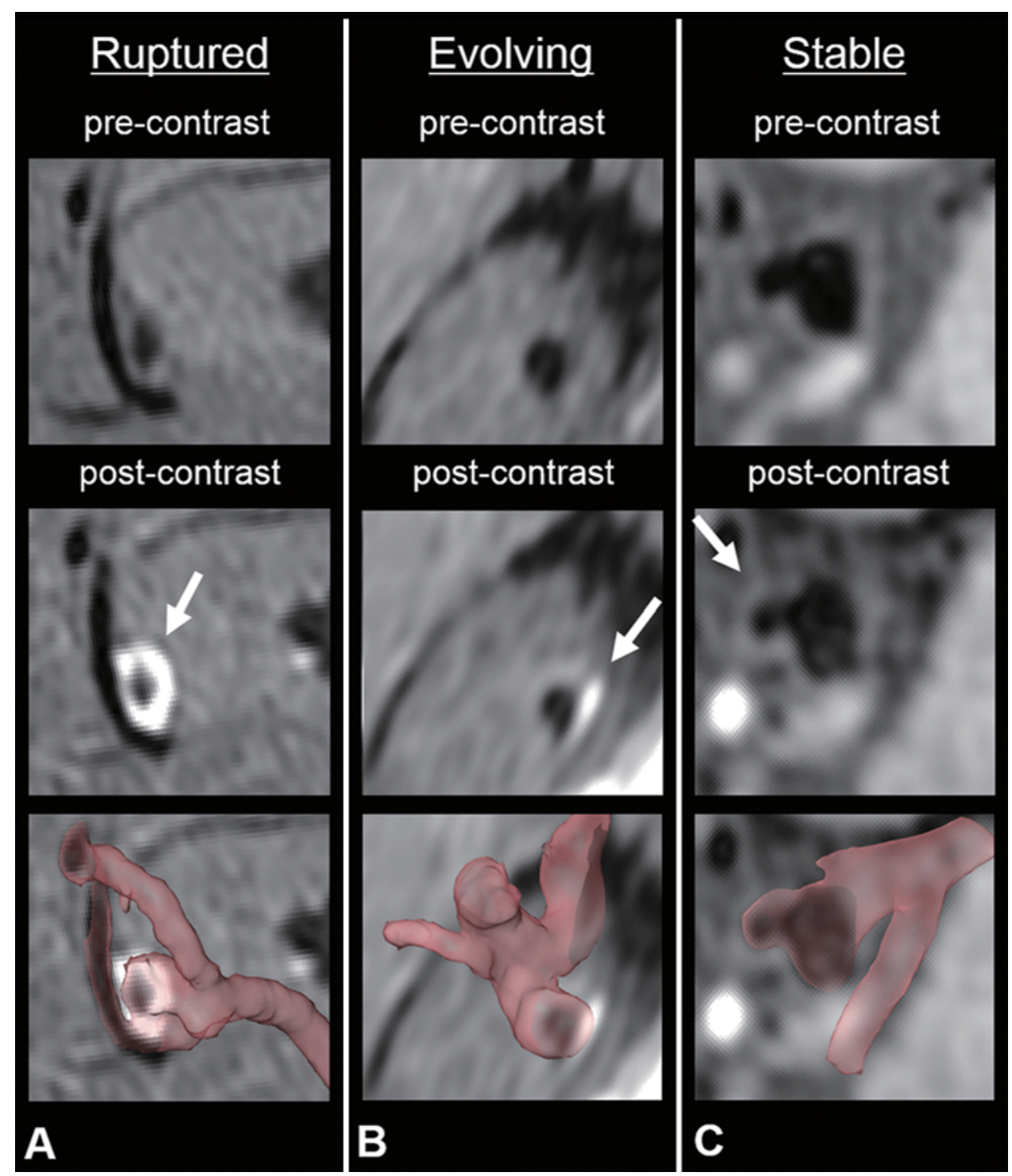

FIG. 3. Cases of ruptured, evolving, and stable aneurysms showing different MR vessel wall imaging findings. The upper, middle, and lower rows show precontrast T1-weighted imaging, postcontrast T1-weighted imaging, and postcontrast T1-weighted imaging superimposed with transparent volume rendering of MR angiography, respectively. A: Images from a 71-year-old woman with a ruptured distal ACA aneurysm. A sagittal section of MR vessel wall imaging shows marked enhancement along the aneurysm wall (arrow). B: Images from a 74-year-old woman with an evolving unruptured left PCoA aneurysm. A sagittal section of MR vessel wall imaging shows relatively strong enhancement along the aneurysm wall (arrow). C: Images from a 72-year-old woman with a stable unruptured left PCoA aneurysm. An axial section of MR vessel wall imaging shows little enhancement along the aneurysm wall (arrow). Figure is available in color online only.

further inflammatory response in the wall, ultimately resulting in aneurysm rupture. ${ }^{7}$ In contrast, CEAW of unruptured aneurysms is considered to be caused by aneurysm wall inflammation and vasa vasorum. ${ }^{6,16}$ Vasa vasorum is also associated with the morphological modification and rupture risk of aneurysms. ${ }^{24}$ The relationship between CEAW and the risk of rupture is gathering much attention, although the relation may be complex and certainly warrants further investigation. The increased CEAW in the evolving aneurysms demonstrated in this study emphasizes that CEAW is indicative of aneurysm wall inflammation and aneurysm instability; thus, unruptured aneurysms with higher degrees of CEAW may be at high risk for aneurysm rupture.

The quantitative measure used in this study permitted an objective assessment of the association of CEAW with aneurysm evolution. This study showed that the most reliable cutoff value of $\mathrm{CR}_{\text {stalk }}$ for differentiation between evolving and stable unruptured aneurysms was 0.39 , with an associated sensitivity of 0.88 and specificity of 0.63 . The overlapping degree of CEAW between evolving and stable aneurysms in this study can be explained as follows. Aneurysms grouped as evolving could have included aneurysms becoming a stable state after an evolving state 
because of their intermittent growth pattern. ${ }^{7,26,30}$ On the other hand, incidentally found stable-grouped aneurysms could also have included aneurysms in the growing period subsequent to the resting period. In other words, the evolving aneurysms might not include all aneurysms that go on to rupture and might not exclude all stable aneurysms, which is one of the limitations imposed by the retrospective design of this study. In addition, an aneurysm wall with atherosclerotic change could have inflammatory activity in various degrees. ${ }^{17,27}$ The degree of inflammation, which can correspond to CEAW, could have been varied in both evolving and stable aneurysms.

We have reported $\mathrm{CR}_{\text {stalk }}$ as a convenient indicator of CEAW in daily practice, which can be obtained from single postcontrast scanning. ${ }^{23}$ Attention should be given to assessing $\mathrm{CR}_{\text {stalk }}$ because structures adjacent to the aneurysms with high intensity on T1-weighted imaging, such as fatty tissues in the skull and hematomas in the methemoglobin phase, could be confusing. In addition, slow flow and turbulent flow can result in a hyperintense signal that may be misinterpreted as enhancement. We excluded large aneurysms $>12 \mathrm{~mm}$ because the intraaneurysmal flow stagnation effect is more prominent in large aneurysms, as determined in our previous studies. ${ }^{22,23}$ Both pre- and postcontrast imaging should be compared to accurately assess $\mathrm{CR}_{\text {stalk }}$, especially in these confusing situations. Because the SI on MRI varies considerably with different parameter settings, the value of $\mathrm{CR}_{\text {stalk }}$ itself cannot be generalized. However, $\mathrm{CR}_{\text {stalk }}$ can be a useful parameter for the management of intracranial aneurysms due to the close relationship between the degree of CEAW and aneurysm status.

Our study has several limitations. First, the sample size was small because this is a single-center study. Second, findings on MR vessel wall imaging were not verified histologically because of the difficulty in obtaining specimens of the aneurysm. Third, we analyzed the data obtained at both 1.5 and $3 \mathrm{~T}$. The signal-to-noise ratio and spatial resolution at $1.5 \mathrm{~T}$ appear to be insufficient for precise evaluation of minute intramural lesions in intracranial arteries. Fourth, aneurysms smaller than $3 \mathrm{~mm}$ were excluded, considering the low quality of image analysis. Finally, we could not assess the direct association between CEAW in unruptured aneurysms and future rupture or growth because of the retrospective study design. In addition, we included only patients who underwent MR vessel wall imaging, which could cause selection bias. A longitudinal prospective study with a larger number of aneurysms using high-resolution MRI would provide more evidence for the association we have indicated in this study.

\section{Conclusions}

We quantitatively compared the degree of CEAW in evolving unruptured aneurysms with stable unruptured and ruptured aneurysms by using a 3D T1-FSE sequence. The CEAW in evolving aneurysms was higher than those in stable aneurysms, and lower than those in ruptured aneurysms. The degree of CEAW may indicate the process leading to rupture of intracranial aneurysms, which can be useful information to determine an indication for surgical treatment of unruptured aneurysms.

\section{References}

1. Backes D, Rinkel GJ, Laban KG, Algra A, Vergouwen MD: Patient- and aneurysm-specific risk factors for intracranial aneurysm growth: a systematic review and meta-analysis. Stroke 47:951-957, 2016

2. Backes D, Vergouwen MD, Tiel Groenestege AT, Bor AS, Velthuis BK, Greving JP, et al: PHASES score for prediction of intracranial aneurysm growth. Stroke 46:1221-1226, 2015

3. Brinjikji W, Zhu YQ, Lanzino G, Cloft HJ, Murad MH, Wang Z, et al: Risk factors for growth of intracranial aneurysms: a systematic review and meta-analysis. AJNR Am J Neuroradiol 37:615-620, 2016

4. Chalouhi N, Hoh BL, Hasan D: Review of cerebral aneurysm formation, growth, and rupture. Stroke 44:3613-3622, 2013

5. de Rooij NK, Linn FH, van der Plas JA, Algra A, Rinkel GJ: Incidence of subarachnoid haemorrhage: a systematic review with emphasis on region, age, gender and time trends. J Neurol Neurosurg Psychiatry 78:1365-1372, 2007

6. Edjlali M, Gentric JC, Régent-Rodriguez C, Trystram D, Hassen WB, Lion S, et al: Does aneurysmal wall enhancement on vessel wall MRI help to distinguish stable from unstable intracranial aneurysms? Stroke 45:3704-3706, 2014

7. Etminan N, Rinkel GJ: Unruptured intracranial aneurysms: development, rupture and preventive management. Nat Rev Neurol 12:699-713, 2016

8. Friedman JA, Piepgras DG, Pichelmann MA, Hansen KK, Brown RD Jr, Wiebers DO: Small cerebral aneurysms presenting with symptoms other than rupture. Neurology 57:1212-1216, 2001

9. Frösen J, Piippo A, Paetau A, Kangasniemi M, Niemelä M, Hernesniemi J, et al: Remodeling of saccular cerebral artery aneurysm wall is associated with rupture: histological analysis of 24 unruptured and 42 ruptured cases. Stroke 35:22872293, 2004

10. Greving JP, Wermer MJ, Brown RD Jr, Morita A, Juvela S, Yonekura M, et al: Development of the PHASES score for prediction of risk of rupture of intracranial aneurysms: a pooled analysis of six prospective cohort studies. Lancet Neurol 13:59-66, 2014

11. Hu P, Yang Q, Wang DD, Guan SC, Zhang HQ: Wall enhancement on high-resolution magnetic resonance imaging may predict an unsteady state of an intracranial saccular aneurysm. Neuroradiology 58:979-985, 2016

12. Inoue T, Shimizu H, Fujimura M, Saito A, Tominaga T: Annual rupture risk of growing unruptured cerebral aneurysms detected by magnetic resonance angiography. J Neurosurg 117:20-25, 2012

13. Kataoka K, Taneda M, Asai T, Kinoshita A, Ito M, Kuroda R: Structural fragility and inflammatory response of ruptured cerebral aneurysms. A comparative study between ruptured and unruptured cerebral aneurysms. Stroke 30:1396-1401, 1999

14. Krings T, Mandell DM, Kiehl TR, Geibprasert S, Tymianski $\mathrm{M}$, Alvarez $\mathrm{H}$, et al: Intracranial aneurysms: from vessel wall pathology to therapeutic approach. Nat Rev Neurol 7:547559, 2011

15. Kubo Y, Koji T, Kashimura H, Otawara Y, Ogawa A, Ogasawara K: Female sex as a risk factor for the growth of asymptomatic unruptured cerebral saccular aneurysms in elderly patients. J Neurosurg 121:599-604, 2014

16. Lehman VT, Brinjikji W, Mossa-Basha M, Lanzino G, Rabinstein AA, Kallmes DF, et al: Conventional and highresolution vessel wall MRI of intracranial aneurysms: current concepts and new horizons. J Neurosurg 128:969-981, 2018

17. Lou X, Ma N, Ma L, Jiang WJ: Contrast-enhanced 3T highresolution MR imaging in symptomatic atherosclerotic basilar artery stenosis. AJNR Am J Neuroradiol 34:513-517, 2013 
18. Macdonald RL, Schweizer TA: Spontaneous subarachnoid haemorrhage. Lancet 389:655-666, 2017

19. Matouk CC, Mandell DM, Günel M, Bulsara KR, Malhotra A, Hebert R, et al: Vessel wall magnetic resonance imaging identifies the site of rupture in patients with multiple intracranial aneurysms: proof of principle. Neurosurgery 72:492-496, 2013

20. Mugler JP III: Optimized three-dimensional fast-spin-echo MRI. J Magn Reson Imaging 39:745-767, 2014

21. Nagahata S, Nagahata M, Obara M, Kondo R, Minagawa N, Sato S, et al: Wall enhancement of the intracranial aneurysms revealed by magnetic resonance vessel wall imaging using three-dimensional turbo spin-echo sequence with motionsensitized driven-equilibrium: a sign of ruptured aneurysm? Clin Neuroradiol 26:277-283, 2016

22. Omodaka S, Endo H, Niizuma K, Fujimura M, Endo T, Sato $\mathrm{K}$, et al: Circumferential wall enhancement on magnetic resonance imaging is useful to identify rupture site in patients with multiple cerebral aneurysms. Neurosurgery 82:638644,2018

23. Omodaka S, Endo H, Niizuma K, Fujimura M, Inoue T, Sato $\mathrm{K}$, et al: Quantitative assessment of circumferential enhancement along the wall of cerebral aneurysms using MR imaging. AJNR Am J Neuroradiol 37:1262-1266, 2016

24. Portanova A, Hakakian N, Mikulis DJ, Virmani R, Abdalla WM, Wasserman BA: Intracranial vasa vasorum: insights and implications for imaging. Radiology 267:667-679, 2013

25. Qiao Y, Zeiler SR, Mirbagheri S, Leigh R, Urrutia V, Wityk $\mathrm{R}$, et al: Intracranial plaque enhancement in patients with cerebrovascular events on high-spatial-resolution MR images. Radiology 271:534-542, 2014

26. Sonobe M, Yamazaki T, Yonekura M, Kikuchi H: Small unruptured intracranial aneurysm verification study: SUAVe study, Japan. Stroke 41:1969-1977, 2010

27. Sugiyama S, Niizuma K, Nakayama T, Shimizu H, Endo H, Inoue $\mathrm{T}$, et al: Relative residence time prolongation in intracranial aneurysms: a possible association with atherosclerosis. Neurosurgery 73:767-776, 2013

28. Thompson BG, Brown RD Jr, Amin-Hanjani S, Broderick JP, Cockroft KM, Connolly ES Jr, et al: Guidelines for the man- agement of patients with unruptured intracranial aneurysms: a guideline for healthcare professionals from the American Heart Association/American Stroke Association. Stroke 46:2368-2400, 2015

29. Vlak MH, Algra A, Brandenburg R, Rinkel GJ: Prevalence of unruptured intracranial aneurysms, with emphasis on sex, age, comorbidity, country, and time period: a systematic review and meta-analysis. Lancet Neurol 10:626-636, 2011

30. Yonekura M: Small unruptured aneurysm verification (SUAVe Study, Japan) -interim report. Neurol Med Chir (Tokyo) 44:213-214, 2004

31. Ziemba-Davis M, Bohnstedt BN, Payner TD, Leipzig TJ, Palmer E, Cohen-Gadol AA: Incidence, epidemiology, and treatment of aneurysmal subarachnoid hemorrhage in 12 Midwest communities. J Stroke Cerebrovasc Dis 23:10731082, 2014

\section{Disclosures}

The authors report no conflict of interest concerning the materials or methods used in this study or the findings specified in this paper.

\section{Author Contributions}

Conception and design: H Endo, Omodaka, Niizuma. Acquisition of data: H Endo, Omodaka. Analysis and interpretation of data: Omodaka, Niizuma. Drafting the article: Omodaka. Critically revising the article: $\mathrm{H}$ Endo. Reviewed submitted version of manuscript: H Endo, Niizuma, Fujimura, Inoue, T Endo, Sato, Sugiyama, Tominaga. Approved the final version of the manuscript on behalf of all authors: H Endo. Statistical analysis: Omodaka. Study supervision: Fujimura, Tominaga.

\section{Correspondence}

Hidenori Endo: Tohoku University Graduate School of Medicine, Miyagi, Japan. hideendo@gmail.com. 